\title{
VIVENCIA DE DISCAPACIDAD POR TRAUMATISMO DE LA MÉDULA ESPINAL Y EL PROCESO DE REHABILITACIÓN ${ }^{1}$
}

\author{
DISABILITY OF EXPERIENCE FOR SPINAL CORD INJURIES \\ AND THE REHABILITATION PROCESS
}

\author{
Luís GONZÁLEZ ECHEVERRÍA* \\ YOCELYN PRICE \\ LuZ ANGÉliCA MuÑoz ${ }^{* * *}$
}

\begin{abstract}
RESUMEN
La discapacidad es un problema de salud que afecta distintas dimensiones en la calidad de vida de las personas. El objetivo del estudio fue describir las vivencias de las personas con discapacidad por lesión medular. Se presenta una investigación de tipo cualitativa de trayectoria etnográfica, utilizando la entrevista, observación participante de Spradley y el interaccionismo simbólico de Mead. El universo se constituyó por adultos discapacitados por traumatismo grave a la médula espinal, en rehabilitación en la Fundación Esperanza Nuestra. La investigación describe la vivencia de tener una discapacidad por daño a la medula espinal, lo difícil que es asumir esta condición y disponerse a la rehabilitación. Los informantes describen etapas en el proceso de rehabilitación; desde una fase marcada por la depresión, hasta alcanzar en los mejores casos la independencia absoluta. La presencia del profesional de enfermería es una constante en los distintos momentos de la trayectoria de aceptación de la discapacidad y rehabilitación que los informantes describen, por lo que cobra importancia el rol de la enfermera y los efectos de sus cuidados. Se observa en la vivencia una ausencia de participación de los centros de salud de atención primaria, por lo tanto es fundamental fortalecer las bases para el desarrollo de una red para la rehabilitación con base en salud familiar y comunitaria.
\end{abstract}

Palabras clave: Enfermería, traumatismo de la médula espinal, personas con discapacidad, rehabilitación.

\begin{abstract}
Disability is a health problem that affects different dimensions of people's quality of life. The object of the study was to describe the experiences of people with disabilities by spinal cord injury. We present a qualitative study, using Spradley's ethnographic interview and participant observation, and Mead's symbolic interactionism. The cultural universe consisted of adults disabled by severe trauma to the spinal cord, under rehabilitation at "Fundación Esperanza Nuestra". Research describes the experience of having a disability for damage to the spinal cord, how difficult it is to assume this condition and be available for rehabilitation. The informants described various stages in the process of rehabilitation going from a phase marked by depression to a phase of complete independence, in some cases. The presence of the nurse is permanent at the various times of the personal journey towards acceptance and rehabilitation described by the informants, highlighting the importance of the role played by these professionals and the effects of their care. A lack of involvement of primary health care centers, is seen in the experience and therefore it is essential to strengthen the foundations for the development of a network for rehabilitation based on family and community health.
\end{abstract}

Key words: Nursing, spinal cord injuries, disabled persons, rehabilitation.

Fecha recepción: 25/11/2009 Fecha aceptación: 29/03/2011

\footnotetext{
${ }^{1}$ Trabajo extraído de la Tesis de Grado: "Vivencia de ser discapacitado por daño a la medula espinal, estudio etnográfico", presentada en el Programa Magíster en Enfermería de la Facultad de Enfermería UNAB.

* Magíster en Enfermería. Docente Facultad de Enfermería Universidad Andrés Bello, Chile, Email: lgonzalez@unab.cl

** Magíster en Enfermería. Profesor asistente Facultad de Enfermería, Universidad Andrés Bello, Chile Email: y.price@ uandresbello.edu

${ }^{* * *}$ Ph. D. Profesora titular de la Facultad de Enfermería, Universidad Andrés Bello, Chile. Email: lmunoz@unab.cl
} 


\section{INTRODUCCIÓN}

Hoy en día se puede señalar que las personas con discapacidad ya no son una minoría invisible en Chile. La Encuesta Calidad de Vida 2004 indica que existirían 3.292.296 discapacitados en el país. Según el Instituto Nacional de Estadísticas, el 12,93\% de la población chilena presenta algún tipo de discapacidad, lo que equivale a aproximadamente a uno de cada ocho chilenos. En efecto, uno de cada tres hogares en Chile tiene algún familiar con discapacidad, y según la Organización Panamericana de la Salud sólo de un 2 a un $6 \%$ de ellos tienen acceso a una forma efectiva de servicios de rehabilitación $(1,2)$.

En nuestra experiencia profesional, hemos percibido que no todos los profesionales de enfermería desean trabajar con personas discapacitadas y que muchos de ellos nunca han tenido la experiencia de cuidar a personas en esta condición. En esta oportunidad la docencia universitaria facilitó el encuentro con las personas con discapacidad a través de las experiencias de los estudiantes en la Fundación Esperanza Nuestra, a lo cual se sumó el interés del autor principal de realizar una investigación en este campo a partir de su interacción y del compartir con estos pacientes y sus familias mientras brindaba cuidados en esta experiencia en los cursos de atención primaria.

La discapacidad ha sido tratada a nivel internacional como un problema de salud relevante para los países latinoamericanos, así la XXIX Asamblea Mundial de la Salud, celebrada en mayo de 1976, adoptó la Resolución 29.35 mediante la cual se acordó la publicación con propósito experimental de la "International Classification of Impairments, Disabilities, and Handicaps" (ICIDH), que se publicó en español como "Clasificación Internacional de las Discapacidades, Deficiencias y Minusvalías" (CIDDM) por la OMS 1983 (3). Esta clasificación fue posteriormente aprobada por la
Asamblea Mundial de la Salud, bajo el título "Clasificación Internacional del Funcionamiento, de la Discapacidad y de la Salud" (CIF), en la Resolución WHA54.21 del 22 de mayo de 2001 y cuyo nuevo acrónimo, CIF (4), se distancia de la anterior clasificación CIDDM. Con este cambio de nombre se ha intentado reflejar el deseo de sustituir la perspectiva negativa de las deficiencias, discapacidades y minusvalías por una visión más neutral de la estructura y de la función, considerando las perspectivas positivas de las actividades y de la participación (5). Por lo anterior existen definiciones o conceptos que es necesario distinguir cuando se habla de las consecuencias que viven las personas tras sufrir un daño en salud. "Si estas consecuencias a nivel del órgano imposibilitaba que el paciente desarrollase sus actividades de rutina, especialmente las actividades de la vida diaria, lo clasificábamos como teniendo una incapacidad. Maciel (6) refiere que si los datos revelaban la existencia de una pérdida social, o sea, aquellas que comprometían sus necesidades básicas, lo clasificábamos como teniendo una desventaja", refiriéndose a la clasificación internacional de la discapacidad.

En Chile se promulgó la Ley 19.284 en enero de 1994 (7), la cual establece las normas para la plena integración social de personas con discapacidad, en ella se establece la responsabilidad del Estado en cuanto a la prevención de la discapacidad y la rehabilitación. A partir de esta normativa se publicó en marzo de 1995 un decreto supremo, que aprueba el reglamento para la evaluación y calificación de la discapacidad, donde se consigna la discapacidad educativa, laboral y de integración social. Aun en la actualidad este marco regulador parecer no ser suficiente para los que viven con una discapacidad.

La situación de la discapacidad, en nuestro país se logra caracterizar en los años 2002-2004, a través del análisis de datos estadísticos obtenidos por diversos estudios. Así, el CENSO nacional de 2002 (8) arrojó 
que eran 334.377 las personas discapacitadas a nivel nacional. La encuesta de caracterización socioeconómica "CASEN" (9) realizada por el Ministerio de Planificación y Cooperación (MIDEPLAN) dio un total de 564.913 personas discapacitadas, y la Encuesta Nacional de Calidad de Vida (ENCAVI) (10), la cual considera un concepto más amplio de la discapacidad, arrojó como resultado un total de 3.292.296 discapacitados, situación similar a la que se presenta en Cuba (11).

Los estudios más recientes en Chile acerca de la discapacidad, y de la integración social, se realizaron el año 2004 por el FONADIS $(12,13)$, los que arrojaron como resultado que sólo el $6 \%$ de la población discapacitada ha tenido acceso a la rehabilitación. Se destaca que el número de personas discapacitadas se concentra entre los 30 y 60 años de edad. Sin duda que al enfrentar esta realidad, se hace aún más necesaria la existencia de centros especializados en rehabilitación, en los cuales se realice un trabajo integral tanto con el paciente como con su entorno, lo que permitirá su recuperación y reinserción más expedita.

La revisión de la literatura apunta a que la rehabilitación es un proceso dinámico orientado hacia la salud, el que permite a la persona enferma o con discapacidad alcanzar su nivel máximo de funcionalidad. Apuesta también hacia una rehabilitación con base en salud familiar y comunitaria, sin desconocer que al adquirir una discapacidad y enfrentar la rehabilitación se atraviesa por diversas reacciones emocionales.

A pesar que el tema de investigación no fue los efectos del traumatismo raquimedular, sino la vivencia de ser y estar con una discapacidad, en varios de los discursos de los informantes se describen procesos relacionados con los efectos del daño medular. Por esta razón, y a fin de que el lector pueda tener una mejor comprensión del fenómeno estudiado, se ha citado a Gibson entre otros (14-16), quien describe los efectos provocados por un traumatismo grave a la médula espinal de esta forma. La interrogante a la cual daremos respuesta en este artículo es la siguiente, ¿Cómo es la vivencia de convertirse en un discapacitado físico secundario a lesión medular?

\section{Objetivo}

Describir la vivencia de un universo cultural que sufre discapacidad secundaria a una lesión grave a la medula espinal, en su forma de pensar, sentir y actuar dentro de este contexto cultural.

\section{METODOLOGÍA}

Tipo de investigación: Utilizamos la investigación cualitativa de trayectoria etnográfica interrogante. La recolección de datos se realizó a través de la observación participante y entrevistas en profundidad. Para realizar el análisis y la interpretación teórica de los discursos se ha adoptado como referente teórico a Laplantine (18), el interaccionismo simbólico de Mead (19), quien explora cómo las personas definen la realidad y cómo sus creencias se relacionan con sus acciones. El interaccionismo está interesado en el estudio de los aspectos internos o de las experiencias del comportamiento humano, para tal efecto, una vez analizadas las entrevistas se realizó la transcripción de las grabaciones de los rasgos culturales, junto a pares que validaron los significados de las palabras y expresiones utilizadas por los informantes, de este modo emergió el tema cultural, definido como grandes unidades de pensamiento que consisten en un número de símbolos integrados dentro de las relaciones de significados, así el tema es develado a través de la inmersión del investigador en el ambiente cultural, a través de los informantes de los escenarios culturales $(19,20)$.

El primer criterio de selección de los informantes fue su condición de discapacita- 
dos físicos por traumatismo grave a la medula espinal (TGME). Luego, con la ayuda de la enfermera coordinadora del centro, quien conocía muy bien a los internos, se procedió a seleccionar seis informantes claves que cumplían con los criterios de selección; ser buenos informantes representativos del universo cultural, adultos de nivel socioeconómico medio, en etapa de productividad y provenientes de familias funcionales, fueron suficientes para saturar las narrativas del cual emergió el tema.

El referencial metodológico de Spradley 1979 (19) permitió ordenar la investigación: conocer la comunidad, compartir con sus integrantes, seleccionar los informantes, fijar las entrevistas, analizar los discursos, identificar los dominios culturales, los temas, manteniendo al investigador en un contacto continuo con la comunidad por espacio de siete meses aproximadamente. De esta manera siempre retornamos a los informantes con más preguntas descriptivas, estructurales y de contraste, paralelamente se realizó discusión con grupos de profesionales que vivían en la misma cultura para verificar nuestra comprensión. El primer nivel de análisis se inicia a partir de las anotaciones de los discursos del cual surgieron categorías de significado cultural. Se pudieron identificar 32 dominios culturales, los que dieron origen a 4 taxonomías (Tabla 1).

Tabla 1. Taxonomía Asumiendo la Discapacidad* .

\begin{tabular}{|l|l|}
\hline Taxonomía & Dominios culturales asociados \\
\hline $\begin{array}{l}\text { 1. El accidente "tuve } \\
\text { evitado" }\end{array}$ & $\begin{array}{l}\text { 1. Forma de percibirse previo al accidente. "Era una persona común y corriente" } \\
\text { 2. Causa de la discapacidad. "Accidentes y violencias" }\end{array}$ \\
\hline $\begin{array}{l}\text { 3. Característica del accidente y/o violencia. Tuve } 20 \text { formas de haberlo evitado" } \\
\text { 4. Etapas de la } \\
\text { vivencia de ser } \\
\text { discapacitado }\end{array}$ & $\begin{array}{l}\text { como es el control de esfínter" } \\
\text { 5. Característica de la estadía en el hospital. "No soportaba más la cama" }\end{array}$ \\
$\begin{array}{l}\text { 1. Forma de enfrentar la discapacidad. "Tuve que cambiarlo todo" } \\
\text { 3. Tipo de sentimiento frente a la discapacidad. "Rabia, impotencia, arrepenti- } \\
\text { 4. Característica de la primera fase. "Difícil y vergonzoso" } \\
\text { 5. Característica de la segunda fase de la rehabilitación. "Se fue viendo como que } \\
\text { podría lograr más" }\end{array}$ \\
$\begin{array}{l}\text { 6. Una razón para rehabilitarse. "Para estar en su casa con su familia" } \\
\text { 7. Una característica de la rehabilitación. "Épocas malas y otras bastante buenas" } \\
\text { 8. Un paso en la rehabilitación. "Convencerme que sí podía" } \\
\text { 9. Tipo de logro en la rehabilitación. "Se manejan mejor... ya lo asimilaron" } \\
\text { 10. Forma de cuidado de sí mismo, "Me autocateterizó" } \\
\text { 11. Rehabilitación. "Lo aprendido...se lo tengo que transmitir a mi familia" } \\
\text { 12. Forma de percibir la rehabilitación total. "Independencia absoluta" } \\
\text { 13. Forma de expresarse acerca del futuro. "El tiempo se encarga de suavizar" }\end{array}$ \\
\hline
\end{tabular}

*Utilizando el referente metodológico de Spradley 1979, se identificaron 32 dominios culturales que incluyeron tipos de sentimiento, opiniones y descripciones, éstos se agruparon en 4 taxonomías, las que dieron origen a dos grandes temas; en esta tabla se muestra un resumen de las dos primeras taxonomías encontradas. 
El universo de estudio se mantuvo inserto en el centro de rehabilitación, constituyéndose este centro en el escenario cultural. La Fundación Esperanza Nuestra es dependiente del Hogar de Cristo, y su propósito es prestar apoyo mediante la rehabilitación a personas discapacitadas físicas, adultos y de escasos recursos. Esta institución, ubicada en la comuna de Maipú, Región Metropolitana, atiende con dos modalidades: internado por periodos cortos y atención ambulatoria. Para estos efectos cuenta con 25 camas, gimnasio terapéutico, salas de terapia ocupacional, sala de estar y recreación, entre otros. El equipo de salud se encuentra conformado por: kinesiólogo, psicólogo, terapeuta ocupacional, fisiatra, asistente social, personal administrativo, auxiliares de enfermería y personal de servicio y por supuesto una enfermera coordinadora. Se resguardaron los aspectos éticos a través de todo el proceso investigativo, se incorporo el consentimiento informado y se contó con la autorización del director de la fundación, considerando las normas éticas en investigación tanto nacionales como internacionales $(21,22)$, cuidando de esta forma la vulnerabilidad de las personas que participaron en la investigación (23). En el tiempo en que se realizó ésta investigación, la Fundación no contaba con un comité de ética, por lo que el trabajo fue aprobado por el actual comité de la Facultad de Enfermería de la UNAB.

\section{RESULTADOS Y DISCUSIÓN}

Al comenzar su discurso, los informantes vuelven atrás en el tiempo para referirse al momento previo al evento desencadenante de la discapacidad, el cual es caracterizado por un accidente o hecho de violencia. Éste es un tiempo en el que se perciben a sí mismos como una persona común y corriente, como un miembro más de esta sociedad, una persona "normal".

\begin{abstract}
"Bueno yo corría, era atleta, pero estábamos practicando ejercicio, de lanzamiento, de jabalina, y un compañero lanzo la jabalina, y... me la enterró en el cuello, o sea fue ahí sin querer, por que en Maipú hay mucho viento, entonces dicen, los que vieron, que el viento desvió el curso de la jabalina".
\end{abstract}

En otra escena, el discurso nos permite ver un problema socialmente reconocido como la violencia en términos de su magnitud e impacto, y un conjunto de percepciones relativas al instinto de conservación de la vida, el instinto de defensa de los suyos y el sentido protector que lleva a la persona a correr riesgos.

"Íbamos caminando por la calle y vino un chiquillo corriendo a avisarme de que el mismo gallo que le había herido a mi sobrino, y mi hermana se estaban encarando... entonces yo voy corriendo por que yo dije le va a llegar a mi hermana, vamos corriendo, sabe que corrí tan fuerte que llegué desorbitada... no sabia que venía harta gente corriendo y estaban todas alegándole, y llego, y empezó hacer con la pistola de alla ' $p a$ ' ca' $y$ disparó a mí... después yo llegué a la UCI me dijeron que tenía una bala incrusta por ahí por la cervical... así sucedió mi accidente por salir a defender a mi sobrino".

En otra narración vemos el ejemplo de un accidente automovilístico, un evento que le sucede a miles de personas, y que en este caso fue causado por el descuido y por el consumo de alcohol, tanto de quien sufre el accidente como de quien lo causa. El sentimiento de culpa es el que aparece en forma primaria al recordar esta experiencia, transformándose así la acción del afectado en una situación que provoca un cuestionamiento de la propia responsabilidad y mayor sufrimiento por un daño causado (24).

"Sé que el muchacho cuando me a atropelló iba con un poco de trago, crucé la calle en bi- 
cicleta vi un camión que venía, alcanzaba a cruzar, venía lejos el camión, entonces pasé, $y$ entonces aparece este vehículo, que era un vehículo chico, y me pegó en el pie derecho... y me tiró para el parabrisas, para la parte de atrás, o sea para ser un vehículo chico yo creo que iba a demasiada velocidad... gente que estuvo ahí vieron que tuve 20 formas de haber evitado el accidente, yo, yo vi que podía haber evitado un poquito más, yo también iba con un poquito de trago".

Destaca en los informantes el reconocer que el riesgo de sufrir un accidente es probable para todas las personas y se atribuye a éste o a la violencia la causa fundamental del traumatismo medular y por ende de la discapacidad. Se consignan como parte de la causa el ser joven, el daño directo a la médula por proyectil u otro instrumento cortopunzante durante una práctica deportiva, un accidente en la vía publica o un enfrentamiento violento, junto con el abuso de alcohol, las drogas, y las acciones temerarias, los que son reconocidos como aspectos que pudieron haber sido evitados. A raíz de esta reflexión, surge en los informantes gran angustia, lo cual implica en sí mismo un sufrimiento y la causa de un dolor que no se representa físicamente, pero como dice Enrici 2002: "la lengua del cuerpo busca un margen mayor de tolerancia, propone romper reglas para generar otras que le permitan hablar con más propiedad de sus dolores" (26).

Los informantes continúan su narrativa señalando el momento en que la expresión de los daños del accidente se hace perceptible, y en que la persona comienza a experimentar y a sufrir los efectos inmediatos del daño medular, describiéndose a sí mismos con expresiones como: "yo era una persona muerta", lo que muestra inicialmente la pérdida del control de la corporalidad y lo vulnerable y sensible del propio cuerpo, dando cuenta de un proceso de duelo anticipado en la persona, del cual más tarde deberán recuperarse.
"Yo ya asumí que ya estoy de las piernas pa" abajo, yo ya no tengo movimiento, en mis manitos tengo un poco de movimiento".

Es así como con posterioridad al accidente la persona en su cuerpo percibe la realidad, experienciando los efectos del daño medular y en una fase más avanzada el reconocimiento de las potencialidades presentes. Sin embargo, al momento inmediato del accidente, cuerpo y mente se describen como una sola cosa: una persona muerta, inerte, sin efecto en el medio, ni dominio de su propio cuerpo. Nuestro universo cultural reconoce después de sufrir el accidente etapas dentro de la vivencia de ser discapacitado:

"La primera etapa es como cuando... como cuando mueres, o como cuando se te muere un paciente, cuando se te muere un familiar los primeros meses igual estás como triste, como con 'depre', como que no quieres nada, y después a medida que pasa el tiempo, bueno yo miraba para el lado y yo veía que otros que estaban mal, no movían ni las, manos, primero es hacerse como la loca, que uno no entiende lo que pasa, tiene siempre la ilusión de que va a volver a caminar, y después es cuando uno se da cuenta, que no va a volver a caminar que es discapacitado, y después viene la otra etapa que es como rabia, impotencia, rabia, enojo..., después viene cuando uno sufre, yo ya asumí, que estoy de las piernas para abajo ya no tengo movimiento, en mis manitos tengo un poquito de movimiento y me han estado trabajando ahora para ver que pasa con ellas, me han estado trabajando las manitos y es como que estoy dando un poquitito más, me siento hasta el pecho más menos y de ahí para abajo no siento nada”.

Luego del accidente la persona es llevada al hospital, donde hace frente a su cuerpo e iniciará una nueva construcción de su yo, en un ambiente extraño y en el cual deberá asumir un rol de paciente. Esto constituye 
una experiencia donde el ser se encuentra totalmente limitado, dependiente de otros y en un momento donde además se enfrenta a sentimientos difíciles de sobrellevar.

Los informantes se ven a sí mismos como vulnerables, con limitaciones y se describen como moribundos. En el plano físico surge el cansancio de estar postrado, y de los efectos de esa misma condición, la producción de úlceras por presión: "No soportaba más la cama". La cama se constituye en el espacio físico donde transcurre y se vivencia toda la lucha por la sobrevivencia y el cuestionamiento del sentido de la vida, la persona se siente privada de la libertad de expresión de su persona a través del cuerpo.

"Para mí ha sido difícil y vergonzoso, pero las chiquillas le dan confianza, por ser aquí hay dos niñas jóvenes, para ser tan joven, ellas me dan la confianza que me puede dar un adulto...luego como que se le quita la vergüenza, pero igual se siente, se siente un poquito mal, con la orina, la orina uno, la orina la hecha aquí, yo ando con sonda foley, la hecha ahí”.

Se hace presente la vergüenza como una turbación del ánimo por alguna acción deshonrosa y humillante propia, esto coincide con lo que dice Laplantine (18) acerca de la enfermedad como humillación: "La enfermedad es vivenciada como infamia, una desgracia, en un momento la persona prefiere la muerte por sobre la disminución de la capacidad física y la presencia de la enfermedad para toda la vida, los discursos parecen representativos de una de las connotaciones más importantes atribuidas al mal-enfermedad en nuestra sociedad: ese mal no es únicamente percibido como una privación, que pide por una exclusión, pero sí como una humillación atroz".

Así, durante esta etapa se perciben fuertemente consecuencias y características del daño medular tales como la tetraplejia, la que se manifiesta en los siguientes términos: "para abajo yo ya ni esperanza tengo". También se evidencia una pérdida del rol social y de la familia ejemplificada en el caso de quienes son madres y se ven imposibilitadas ya de asumir la responsabilidad de atender a los hijos. Además, se deben enfrentar síntomas físicos como espasmos musculares y la pérdida del control de esfínter, los informantes señalan que esta situación los impacta en forma íntegra, lo cual describen diciendo: "viene el paquete completo", y que como resultado hace que esta experiencia sea descrita como algo "difícil y vergonzoso".

La persona, al sentirse viva, comienza a buscar formas de enfrentar la discapacidad, refiriéndose a distintas actividades que deben realizar, y a cambios que exceden en muchos casos las expectativas, lo señalan cuando dicen: "tuve que cambiarlo todo", esta actitud, esta acción es la que les permite adaptarse a la nueva forma de vida. La experiencia de vivir con una discapacidad es un fenómeno que se vive desde el interior hacia el exterior, la persona, estando lúcida y en pleno juicio, debe hacer una valoración de sí misma e intentar resolver una serie de preguntas tales como ¿por qué yo?, ¿para qué?, ¿qué hay del futuro? y ¿cuál puede ser mi aporte en esta vida? Nuestro universo cultural describe una gran crisis vivencial, que gatilla un estado de depresión profunda, en el que la persona debe lidiar con su realidad, y poner en juego su familia, su fe o su filosofía de vida, y son justamente sus seres queridos y la fe personal los que empujan a la persona a disponerse a la rehabilitación. De esta manera, vemos en el discurso de nuestros informantes que para pasar a una segunda fase la persona debe convencerse a sí misma que es posible vivir con una discapacidad. Para esto debe reconocer sus redes de apoyo, sentir el respaldo de sus seres queridos, realizar la búsqueda de respuestas y fortalecerse en la fe. El universo cultural inicia esta segunda fase con un periodo de reflexión, en donde se identifican las razones por la cuales seguir viviendo y realizar la rehabilitación. Estas razones son 
diversas y dependen de los contextos en los cuales cada persona se encuentra:

"Lloré un poquito, quedé un poquito, achacá que uno está, pero yo digo no por mi hijo tengo que tirar pa' arriba".

"Entonces a veces yo miraba y decía, igual estoy puro 'tonteando', si yo tengo mis manos buenas mi cabeza buena, igual puedo tirar para arriba, igual puedo hacer cosas".

Este discurso evidencia un proceso reflexivo más individual, tiene que ver con el enfrentamiento a la discapacidad, la necesidad de recuperar la independencia, y fortalecer las capacidades remanentes y las potencialidades intactas del cuerpo. Es un discurso que además nos muestra una posición más pragmática y sólida, en cuanto se relaciona con la autodeterminación a realizar una tarea, siendo más cercano a la acción en sí misma.

Otro discurso nos muestra la experiencia misma del encuentro con actores sociales que se van incorporando al escenario de la realidad del paciente, lo que permite dimensionar la importancia de la acción social, del estar con otros y de determinar las acciones propias en el encuentro con ellos.

"A mí en lo personal me he sentido... como que he sentido una misión, de alguna manera, tú sabes, el hecho de que el Padre Aldo de alguna manera, él era un discapacitado también, tenía una tetraplejia también y tú sabes, el hecho de que él era tetrapléjico, yo era tetrapléjica, y él como que me habló de su obra, de lo importante que los discapacitados nos uniéramos y lucháramos por nuestros derechos".

En consonancia con Laplantine (18), "existe un segundo nivel de enfermedad, la enfermedad-proeza: es inverso a la enfermedad humillación, es a partir de una experiencia patológica, una enfermedad, un accidente, la persona da pruebas de una vo- luntad excepcional, ampliando al máximo sus virtualidades que él probablemente no desarrollaría, sin esa revelación constituida, en el modelo de enfermedad". El sentido que la persona le da a la vida es fundamental al momento de rehabilitarse, pues ahí se fundamenta su razón para seguir existiendo y ser un aporte para sus seres queridos.

Al racionalizar su discapacidad la persona identifica las razones que ya hemos revisado, aun cuando para lograr estos propósitos la persona debe tener como meta la autovalencia, es decir, estar en las mejores condiciones físicas y sicológicas posibles, para no ser una carga para su familia, ni depender de un servicio sanitario, ni para dejar de ser una persona productiva, todo lo cual se plantea en términos de un objetivo a perseguir: "una labor, una misión".

La rehabilitación se inicia de forma muy característica, en un centro de rehabilitación en donde los pacientes cuentan con apoyo interdisciplinario. En este escenario ellos van describiendo su rehabilitación a través de los logros que obtienen, lo que provoca cambios en su interpretación del mundo y de las relaciones con los demás. Los sentimientos que a raíz de ello experimentan son diversos y el cambio en su propia forma de enfrentar al mundo y su discapacidad se torna en lo más relevante.

"No podía hacer nada, movía los dedos cuando necesitaba algo, $y$ ahora usted me ve... me muevo, me paro, hago cucharitas, ya estoy haciendo traspaso a la tabla, de la silla a la cama, y de ahí viene el traspaso de la silla a la tasa del baño, que es bueno, me baño, "me han ido haciendo las terapias pa" las manitos, porque me han hecho ejercicios, participamos en juegos".

Se observa cómo la rehabilitación desde su inicio se relaciona con volver a tener control del cuerpo, el fortalecimiento de capacidades que más adelante les permitirán realizar su autocuidado, se trata de pequeños 
logros que los incentivan a seguir adelante en este proceso.

Gibson (15) refiere que "tras sufrir un TGME, la mayoría de los pacientes empieza a conocer su cuerpo de manera muy detallada. $\mathrm{Al}$ escuchar con cuidado al paciente, la enfermera puede conocer no sólo sus síntomas actuales sino también lo que el paciente considera como normal. A menudo, los pacientes con TGME desarrollan una serie de rutinas diarias, como por ejemplo, el momento en el que realizan los ejercicios de la gama de movimientos o en el que llevan a cabo el programa intestinal. Las intervenciones de enfermería se deben programar con el mayor ajuste posible a las rutinas del paciente".

De esta manera, los sentimientos que se presentan en este momento van a la par con pequeños logros que la persona va experimentando, siendo el requisito fundamental que ellos describen para lograr avanzar es disponerse y "convencerse que se puede vivir con una discapacidad". En la medida que se da este convencimiento, esta certeza que pueden vivir con discapacidad, nuestro universo cultural también inicia una búsqueda de terapias u otros tratamientos que le permitan llegar a ser autovalentes de la mejor forma posible. Dentro de ellas destaca la cirugía, la que, aunque los ayuda, no será siempre por sí sola la que los llevará a su meta. Sin duda, ellos mantienen en la mente su recuperación, y eso los lleva muchas veces a no reflexionar sobre posibles complicaciones de las operaciones o dilataciones en su rehabilitación a causa de este tipo de procesos.

"Un doctor bien bueno, bien cordial para conversar, profesional, yo creo que él vio mi desesperación, porque yo ya no aguantaba más sin operarme, claro que él me operó, y después yo perdí más la fuerza... o sea no se mejora al tiro, y aquí estoy tratando de recuperarla, porque yo ahora recién estoy haciendo pesas y cosas, y no pensé que iba a ser tan difícil, porque decía yo opero y después te vas para tu casa, pero me mandó para acá, el mismo doctor me mandó para acá, porque sabía que en la casa habría sido demasiado difícil".

Es posible que al estar con otras personas con distintos grados de discapacidad se promueva la participación de cada una ellas en la propia rehabilitación, las personas pueden observar la evolución de otras con más experiencia, ellos visualizan en otros los tipos de logros en la rehabilitación: "se manejan mejor... ya lo asimilaron". Nuestros informantes expresan así la importancia de la rehabilitación con grupos de pares: "yo miraba al puro compañero, mirándolo a él aprendí", haciendo presente "el mi" aprendiz en sociedad. Inicialmente, se observan a sí mismos con menos desarrollo de las capacidades en comparación a los compañeros que tienen más tiempo en el proceso de rehabilitación, el mirar a los otros y compartir con ellos les impone un desafío, una meta a alcanzar, manejarse mejor, adquirir destrezas tales como el autocateterismo vesical, el traslado de la cama a la silla, el vestirse solos, etc.., y finalmente asimilarlo, es decir, esta experiencia, se transforma en una instancia de cambio, una acomodación en la forma del diario vivir, asumiendo la discapacidad en forma natural, sin impedimentos para el desarrollo personal.

Los resultados iniciales de la rehabilitación se relacionan con la actitud del sujeto frente a la misma. Sólo cuando las personas logran centrarse en el aquí y en el ahora y cuando reconocen ciertas capacidades como el estar bien y sentirse lúcido -lo que verbalizan en expresiones como "tengo mis manos buenas mi cabeza buena" о "uno mismo se va conociendo"- es cuando se dan las bases relacionadas con la voluntad, la libertad psicológica y emocional. Es con esta idea en mente que la persona permanece en el camino de la rehabilitación a pesar de las adversidades, pero reconociendo sus logros, los que son representados en sus discursos como una situación de menor enfermedad: 
"el sistema inmunológico se fortalece". A esta altura reconocen que la rehabilitación es un proceso más bien lento, difícil, desde el que puede mirar el futuro con optimismo, y en el que también pueden comenzar a fortalecer su identidad personal. Aquí nuevamente aparece "el mi" como tomador de un rol para fortalecer "el yo". Uno de los informantes señaló que en su caso padeció mayormente de enfermedades respiratorias precisamente mientras más protegido estuvo en su casa y que, por el contrario, que a mayor exposición e independencia constató un fortalecimiento de su organismo, razón por lo cual estima que en su experiencia existieron problemas de inmunidad:

"De repente un resfriado que son un poco complicado, las presiones, el ser discapacitado, trabajar más, el sistema inmunológico en mi caso se fortalece, por que yo tuve mucho más bronconeumonias cuando estuve en casa sin hacer nada... tres hospitalizaciones graves... ahora que he salido media mojada con frío con cambios de temperatura, no pasa nada, yo creo que se fortalece un poco el sistema inmunológico".

Así, en esta fase se muestran un poco más tranquilos, y se abren a todas las posibilidades de rehabilitación, pero esta vez de una forma mucho más objetiva, facilitando así la expresión de su propia vivencia y la posibilidad de compartirla con los familiares y el equipo de salud, lo que señalan diciendo: "empecé a hablar como más de mis cosas". Descubren además, y como parte de una etapa de aceptación que pueden lograr otras cosas: "se fue viendo como que podría lograr más", por lo cual se trata de una aceptación activa en que la persona acelera su proceso de rehabilitación.

Sin duda, la rehabilitación pasa a ser un proceso individual, el cual no avanza al mismo ritmo o a la misma velocidad para todas las personas, ya que sólo después de haber transcurrido varios años es cuando se logra o se está muy cerca de una rehabilitación total. Los informantes reconocen como una característica propia de la rehabilitación, el transitar por periodos estresantes tales como complicaciones secundarias a la dismovilidad y el aprendizaje de técnicas para su autocuidado y autovalencia, con ello deben enfrentar además una serie de complicaciones psicosociales asociadas a la discapacidad física: problemas económicos familiares, problemas para el desplazamiento en la ciudad, para relacionarse y establecer relaciones de pareja, entre otros.

"He superado todo, ésta es la etapa como más larga, pensando como persona, trabajar donde me gusta, hacer lo que quiero, es como una tercera etapa". En esta etapa, las personas se describen ciento por ciento rehabilitadas, sienten que ya han pasado por todos los eventos relacionados con el hecho de vivir con una discapacidad y que los han podido superar. El dolor, vivenciado como dolor total, psicológico, espiritual, producido por las pérdidas y las complicaciones que produjo la discapacidad, son parte de sus historias de vida y, en cuanto a ello, señalan: " $E l$ tiempo se encarga de suavizar", es así como se evidencia que las personas necesitan de un gran periodo de tiempo para darle sentido a la vida, superar las frustraciones y disipar el dolor.

Los informantes refieren que el vivir con una discapacidad física significa vivenciar épocas malas y otras bastante buenas, las "épocas buenas" son aquellas en que se experimentan logros, manejo de técnicas que proporcionan mayor independencia, periodos donde no se producen complicaciones tales como las enfermedades respiratorias, y finalmente perciben la rehabilitación total como el logro de la independencia absoluta. Después de un largo camino y de haber adquirido varias destrezas y una actitud permanente de cuidado de sí mismo y autovalencia, la persona se siente rehabilitada totalmente, señalando como característica principal de esta etapa el no depender de 
terceras personas.

Con respecto al futuro, las personas hacen dos tipos de reflexiones que se centran en la incertidumbre, así en primer lugar expresan preocupación por su familia y el futuro de sus seres queridos, el temor frente a la posibilidad de no poder llegar a las metas propuestas o incluso no recuperar la posibilidad de cumplir con un rol social determinado, como contraparte se presenta una reflexión que los lleva a una visión optimista donde se observan con menos sufrimiento, haciendo una vida lo más normal posible y minimizando los efectos del accidente.

La rehabilitación no sería posible sin la existencia de las redes de apoyo, el grupo de pares, la familia o algún ser querido, los incentivos de la fe o la filosofía de vida y la participación de un equipo profesional y técnico multidisciplinario, altamente comprometido con su rol social, como lo es la Fundación Esperanza Nuestra, como también nos muestra la evidencia de otras en casas de rehabilitación (26).

\section{CONSIDERACIONES FINALES}

$\mathrm{Al}$ obtener los resultados finales e interpretarlos a través del interaccionismo simbólico, se logró comprender la vivencia de la persona con discapacidad secundaria por una lesión grave a la médula espinal, en su forma de pensar, sentir y actuar dentro de este contexto cultural, y se pudo obtener aún más descripciones que no se habían contemplado antes, pudiendo realizar un mapa cronológico de los acontecimientos, experiencias vividas, y una descripción etnográfica y fenomenológica que fue más allá de lo que los investigadores habían delimitado como el escenario cultural, utilizando discursos que describían la estadía en hospital, en el hogar y el centro de rehabilitación.

Las limitaciones del estudio se evidencian en los discursos de los informantes que no hacen alusión a cuidados o intervenciones desde la atención primaria en salud, lo que demuestra que en este grupo cultural los centros de atención primaria se encuentran ausentes. Por otra parte, la participación de los investigadores se limitó sólo a la Fundación Esperanza Nuestra, por lo que la investigación se centró en el fenómeno vivido, pero sin observaciones de campo en el núcleo familiar, lo que deja un campo de interrogantes para nuevas investigaciones en el tema.

Los resultados de esta investigación son coherentes con otros autores tales como Laplantine (18), quien identifica que el sentido que la persona le da a la vida es fundamental al momento de rehabilitarse, y Gibson (15), quien aconseja que las intervenciones de enfermería se deben programar con el mayor ajuste posible a las rutinas de cada paciente.

Destaca el dominio cultural "difícil y vergonzoso", el cual desde un punto de vista antropológico es un fenómeno social, cuando los discapacitados muchas veces se sienten mirados con lástima, situación que los incomoda (27), lo que lleva a que la vivencia de la discapacidad está condicionada en gran parte por la sociedad; los informantes lo consignan claramente: "la sociedad y la ciudad misma no están adaptadas", en consonancia con lo referido por Rueda: "el discapacitado pasa a constituirse en un ser humano más vulnerable, lo que hace necesario adoptar medidas especiales -desde un punto de vista éticopara proteger sus derechos como persona, ante decisiones que lo afectan en el ámbito de la salud" (24).

Para ellos no todo es negativo, tanto en el discurso como en las observaciones de campo se advierte un grupo organizado, con identidad cultural, según Mead (19) la persona tendría la capacidad de ser creativo socialmente, adaptándose al medio, convirtiéndose en alguien distinto, pero al hacerse un individuo diferente ha afectado a la comunidad en que vive, la gente tiene que adaptarse a él tanto como él se adapta a ella, y la misma sociedad cambia en ese proceso 
y se convierte, hasta cierto punto, en una sociedad diferente.

Para Alvarado et al. (29), el trabajo de reinserción social es una ardua labor, “... a través del proceso se van obteniendo logros importantes, que se espera en el tiempo sigan su curso y se asuman como propuestas colaborativas de interdependencia y de formación de redes; el proceso de construcción de comunidad para lograr la tan ansiada autonomía, propia de la inclusión social, debe tomarse con cierta reserva en la medida en que en la evaluación siguen persistiendo expresiones que connotan la necesidad de la presencia del otro, desde el vínculo formado en la interacción" $(30,31)$.

El profesional de enfermería se encuentra presente en cada una de las fases de la experiencia de rehabilitación, en la comunidad, en el hospital y en el centro de rehabilitación. Es tarea de este profesional comprender a la persona, considerando su entorno, su cultura y su subjetividad, con el objetivo de establecer una relación de ayuda efectiva. Considerando la realidad de nuestro entorno físico y cultural, las limitaciones y las potencialidades descubiertas a través de la investigación, se puede decir que es fundamental fortalecer las redes de apoyo para la rehabilitación, es decir afianzar las bases para el desarrollo de una red que asegure la rehabilitación en la comunidad donde la persona experimenta sus vivencias.

La rehabilitación con base comunitaria tiene como objetivo "poner a la disposición de las personas con discapacidad y sus familiares de forma racional y eficiente los recursos materiales y humanos disponibles; la familia y los miembros de la comunidad", según lo define la OIT, UNESCO, OMS, RBC, $2004(32,33)$. Por lo tanto es fundamental fortalecer las bases para el desarrollo de una red para la rehabilitación con base en salud familiar y comunitaria, y que la presencia del profesional de enfermería, como un actor reconocido por la comunidad, cobre importancia en las relaciones interpersonales, en el cuidado y en futuras investigaciones.

Agradecimientos a Lilian Garín, enfermera coordinadora de la Fundación Esperanza Nuestra, Maipú, Santiago de Chile.

\section{REFERENCIAS}

1. Vásquez A. Discapacidad: Una perspectiva desde los derechos humanos y la rehabilitación de base comunitaria (RCB). Conferencia. Universidad Andrés Bello, Viña del Mar, Chile; 2009.

2. Torres A, Sanhueza A. Modelo estructural de Enfermería de calidad de vida e incertidumbre frente a la Enfermedad. Cienc. enferm. 2006; 12(1): 9-17.

3. Organización Mundial de la Salud. Clasificación Internacional de Deficiencias, Discapacidades y Minusvalías. Manual de clasificación de las consecuencias de la enfermedad. Madrid: Instituto Nacional de Servicios Sociales; 1983.

4. Chaná P, Alburquerque D. La clasificación Internacional del Funcionamiento, de la discapacidad y de la Salud (CIF) y la práctica neurológica. Rev Chil NeuroPsiquiat. 2006; 44(2): 89-97.

5. Jiménez M, Paulino D, Martin J. La clasificación internacional del funcionamiento, de la discapacidad y de la salud (CIF) 2001. Rev Esp Salud Pública. 2002; 76(4): 271-279.

6. Maciel G. Deficiências, incapacidades e desventagens decorrentes de causas externas -análise em pacientes internados no IOT-HCFMUSP. $28 \mathrm{~cm}$ [tesis doctoral]. Escola de Enfermagem da USP. I Acidentes e Violências - Seqüelas, II Titulo: São Pablo; 1991.

7. Ley de Integración Social de las Personas con Discapacidad. Ley No19.284. Reglamentación para la evaluación y clasificación de la discapacidad. Decreto Ley 250, 
Santiago de Chile, 1994. Biblioteca del Congreso Nacional; 1994.

8. Instituto Nacional de Estadísticas. Comisión Nacional del XVII CENSO de Población y Vivienda "Síntesis de Resultados". Chile; 2003.

9. Ministerio de Planificación Chile, División Social. Análisis de la VIII encuesta de caracterización socioeconómica nacional (CASEN 2000). Documento No 13: Situación de las personas con discapacidad en Chile 2000. Santiago, Chile: MIDEPLAN; 2001.

10. Ministerio de Salud. Primer estudio nacional de la discapacidad, ENDISC-Chile 2004, Informe ejecutivo de resultados; 2005.

11. Cobas M, Zacca E, Morales F, Icart E, Jordán A, Valdés M. Caracterización epidemiológica de las personas con discapacidad en Cuba. Rev Cub Salud Publica [Revista enla Internet].2010; 36(4): 306-310. Hallado en: http://scielo.sld.cu/ scielo.php?script=sci_arttext\&pid=S0864$34662010000400004 \& \operatorname{lng}=$ es. Acceso el 27 de abril 2011.

12. FONADIS. FONADIS. Primer estudio nacional de la discapacidad en Chile. ENDESIC-CIF 2004. Resultados Informe ejecutivo XI Región Aysén 2004 [Internet]. Santiago, Chile; 2005. Hallado en:http:// www.fonadis.cl/centro/estudios endisc2004.php. Acceso el 18 de octubre 2010.

13. Primer estudio nacional de la discapacidad en Chile ENDESIC. Resultados Informe ejecutivo XI Región Aysén 2004. Hallado en: http://www.fonadis.cl/centro/ estudios_endisc2004.php. Acceso el 18 de octubre 2010.

14. García D. ¿Puedo conducir un automóvil, doctor?: Evaluación para el manejo de automóviles en personas con discapacidad y su importancia en la rehabilitación. Rev. Med Chil. 2010; 138(2): 243-250.

15. Gibson K. Cuidados al paciente que vive con una lesión de la médula espi- nal. Nursing: edición española [Revista en Internet] 2004; 22(2): 8-13. Hallado en: http://dialnet.unirioja.es/servlet/ articulo? codigo $=2479174$. Acceso el 18 de octubre 2010.

16. Leal MB Filho, Borges G, Almeida BR, Aguiar A, Vieira M, Dantas K et al. Spinal cord injury: epidemiologycal study of 386 cases with emphasis on those patients admitted more than four hours after the trauma. Arq. Neuro-Psiquiatr. 2008; 66(2b): 365-368. Hallado en: http://www. scielo.br. Acceso el 27 de abril 2011.

17. Rabeh S, Caliri M. Functional ability in individuals with spinal cord injury. Acta paul. enferm. 2010; 23(3): 321-327. Hallado en: http://www.scielo.br. Acceso el 27 de abril 2011.

18. Laplantine F. Antropología da doença. Sao Paulo: Martins Fontes; 1991. p. 116117.

19. Mead G. Espíritu, persona y sociedad; desde el punto de vista del conductismo social. Ed. Paidós. 1990.

20. Spradley J. The Ethnographic Interview. Holt, Rinehart and Winston. U.S.A. 1979.

21. Spradley J. Participant Observation. Holt, Rinebart and Winston, 1980.

22. Consejo de Organizaciones Internacionales de las Ciencias Médicas (CIOMS), Organización Mundial de la Salud. Pautas Éticas Internacionales para la Investigación y Experimentación Biomédica en Seres Humanos [Internet]. Ginebra; 2002. Hallado en: http://www.paho.org/ Spanish/BIO/CIOMS.pdf. Acceso el 27 de marzo 2011.

23. Sotomayor M. Regulación de la investigación biomédica en Chile. Acta Bioeth. [Revista en Internet]. 2008; 14(1): 79-89. Hallado en: http://www.scielo.cl. Acceso el 27 de abril 2011.

24. Rueda L, Miranda O. Principales dilemas bioéticos en las personas con discapacidad prolongada. Acta Bioeth. 2002; 8(1): 127-135.

25. Muñoz LA, Price Y, Reyes M, Ramírez 
M, Costa M. Vivencia de la depresión del Adulto Mayor en los cuidadores familiares. Rev Esc Enferm USP. 2010; 44(1): 32-39.

26. Enrici A, Alvares P. El cuerpo denuncia. Aporte para una Lectura Hermenéutica de la auxología en la encrucijada de la multiculturalidad. En III Congreso Nacional de Investigaciones en Antropología de Perú. Argentina, Universidad Nacional de la Patagonia Austral; 2001.

27. Santos J, Silveira M. Health-care practices performed at assisted living facilities: pursuing the paths back to society. Rev Esc Enferm USP. 2009; 43(4): 788-795.

28. Rodríguez E, Martínez J, Díaz A, Ros A, Abreu Y. Impacto de la Fisioterapia para la reeducación del suelo pélvico en la calidad de vida de pacientes con incontinencia urinaria. Rev haban cienc méd [Revista en la Internet]. 2009; 8(3). Hallado en: http://scielo.sld.cu/scielo. php? script $=$ sci_arttext\&pid $=S 1729$ 519X2009000300013\&lng=es. Acceso el 27 de abril 2011.

29. Alvarado A, Moreno M, Rodríguez M. Inclusión social y participación comunitaria: Una perspectiva de trabajo frente a la discapacidad. Cienc. enferm. 2009;
15(1): 61-74.

30. Rodríguez M, Alvarado A, Moreno M. Construcción participativa de un modelo socioecológico de inclusión social para personas en situación de discapacidad. Acta Colombiana de Psicología. 2007; 10 (2): 181-187.

31. Márquez A. Rehabilitación Basada en la Comunidad en la Costa Pacífica. Revista Fundamento. 2002; 13: 44-53.

32. OIT, UNESCO, OMS, RBC. Estrategia para la rehabilitación, la igualdad de oportunidades, la reducción de la pobreza y la integración social de las personas con discapacidad. 2004. Hallado en: http://www.ilo.org/skills/what/pubs/ lang--en/contLang--es/docName-WCMS_107943/index.htm. Acceso el 27 de abril 2011.

33. Nabergoi M, Bottinelli M. Discapacidad, pobreza y sus abordajes: Revisión de la estrategia de rehabilitación basada en la comunidad (RBC). Anu Investig (Fac. Psicol. Univ. B. Aires) 2007 [Revista en la Internet]. Hallado en: http://www.scielo.org.ar/scielo. php?script=sci_arttext\&pid $=$ S1851$16862007000100015 \& \operatorname{lng}=$ es. Acceso el 27 de abril 2011. 